\title{
P124 Impact of Tapering on Arterial Blood Pressure Using a One-dimensional Computational Model
}

\author{
Shima Abdullateef ${ }^{1 *}$, Jorge Mariscal-Harana ${ }^{2}$, Jordi Alastruey ${ }^{2}$, Ashraf Khir ${ }^{1}$ \\ ${ }^{1}$ Biomedical Engineering Research Theme, Brunel University London, Middlesex, United Kingdom

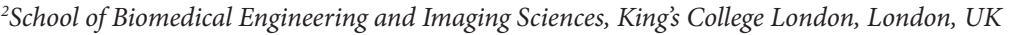

\section{ABSTRACT}

Aortic tapering is a known characteristic of the arterial tree affecting the development of pressure in the aorta. With tapering, the cross-sectional area of vessels decreases moving towards the periphery causing reflections to travel back to the heart. The reflection waves present in the aorta are an amalgamation of reflections from tapering, bifurcations, and mismatched mechanical properties. The difference between the contribution of tapering and of bifurcations or mechanical properties in the overall reflection is not well understood. The aim of this study is to evaluate the impact of tapering, solely, on pressure and wave intensity. We simulated the blood flow using a one-dimensional model which solves the conservation of mass and momentum equations in the time domain. The model consists of the thoracic aorta connected to a symmetrical iliac bifurcation and threeelement Windkessel models as the terminal boundary conditions [1]. Different tapering angles $(\theta)$ in the physiological range, up to 1.5 degrees [2], are implemented by changing the diameter at the outlet of the aortic vessel. The area ratio between the parent and the daughter vessels and the mechanical properties are kept constant which allows for investigating the effect of tapering. As shown in Figure 1 (middle), increased tapering angle is associated with an increase in mean blood pressure due to the higher resistance in the segment, and an increase in pulse pressure due to the reduced arterial compliance. Both forward and backward compression wave amplitudes rise with the tapering angle due to higher resistance of the arterial vessel.

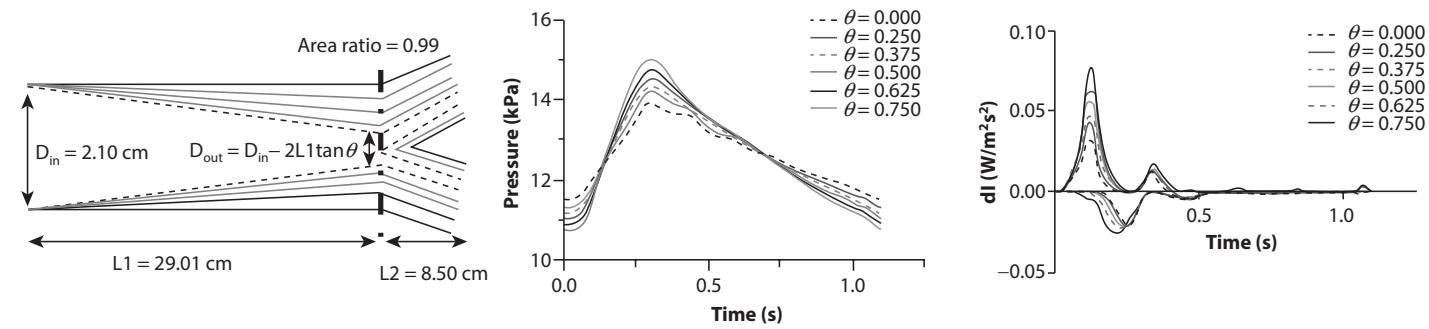

Figure 1 Schematic representation of the thoracic aorta and iliac bifurcation model (left), pressure waveforms at the inlet of the thoracic aorta (middle), and wave intensity analysis at the inlet of the thoracic aorta (right) with different tapering angles.

\section{REFERENCES}

[1] Alastruey J, Parker KH, Peiró J, Sherwin SJ. J Eng Math 2009;64:331-51.

[2] Segers P, Verdonck P. J Biomech 2000;33:299-306.

(C) 2019 Association for Research into Arterial Structure and Physiology. Publishing services by Atlantis Press International B.V. This is an open access article distributed under the CC BY-NC 4.0 license (http://creativecommons.org/licenses/by-nc/4.0/). 